\title{
Autonomous Opportunistic Spectrum Access in Distributed Femtocell Networks
}

\author{
Ahmed Khattab, Khaled Elsayed \\ Electronics and Electrical Communications Department, Cairo University, Giza, Egypt \\ $\{$ akhattab,khaled\}@ieee.org
}

\begin{abstract}
Femtocells randomly deployed in a given macrocell coverage area share the wireless spectrum available to the macrocell. The unplanned and ad hoc nature of the femtocell deployment in the macrocell environment renders centralized frequency planning inapplicable. Furthermore, the femtocells are unable to apriori know neither the channel assignment of their neighbours nor the impact of their transmissions on nearby macrocell users due to the lack of explicit coordination. In this paper, we present the distributed Autonomous Opportunistic Channel Access (AOCA) framework that allows the femtocells to share the available spectrum with the overlaying macrocell without any kind of coordination neither between the macrocell and femtocells nor amongst the femtocells. Furthermore, it provides statistical guarantees on the performance of the macrocell users. We formulate the AOCA constrained spectrum access problem as non-linear program to find its optimized parameters' settings. Simulation results show that AOCA achieves multiple folds improvement in the average femtocell network rate due to its probabilistic and non-greedy access that enables multiple neighbouring femtocells to simultaneously use a given channel.

Index Terms - femtocell networks; opportunistic spectrum access; spectrum sharing
\end{abstract}

\section{INTRODUCTION}

Femtocells - also called home base stations - have recently emerged as a cost-effective solution to improve the indoors coverage and capacity of cellular networks. Typically, femtocells are deployed and managed by customers at homes or in their offices. Therefore, the deployment of femtocells in a preexisting cellular network environment is uncontrollable and results in an unplanned ad hoc femtocell network that shares the wireless spectrum with the macrocell network [1], [2]. Unfortunately, having a dedicated spectrum for the femtocell network to eliminate the interference between the macrocell and femtocell networks (e.g., see [3]-[6]) is typically not feasible due to (1) the scarcity of the wireless spectrum, and (2) the lack of coordination between the macrocell and femtocells and between the femtocells themselves [1], [2]. Hence, it is more favorable to operate the macrocell and femtocell networks in a shared-spectrum manner from either an infrastructure, cost or spectrum availability perspectives [7].

Several approaches have recently emerged to address spectrum sharing in such a two-tier femtocell networks given that centralized network planning is increasingly less viable. A great deal of the literature focuses on the design of power control and interference mitigation strategies [8][10]. Cooperative [11] and non-cooperative [7], [12], [13] gaming techniques have also been widely used to address

This work is part of the $4 \mathrm{G}++$ project supported by the National Telecom Regulatory Authority of Egypt. the spectrum-sharing problem. However, such games require message exchange and coordination between the femtocells, for instance for interference price bargaining, which further deteriorates the attainable femtocell network throughput.

In contrast, our goal is to allow the femtocell network to share the macrocell spectrum by having the individual femtocells locally choosing their transmission bands and strategies without explicit coordination. The paper contributions are:

First, we present the Autonomous Opportunistic Channel Access (AOCA) framework that allows the femtocell network to share the macrocell spectrum while providing statistical guarantees on the performance of the macrocell users. The proposed framework tackles the infeasibility of frequency planning and the lack of explicit spectrum allocation coordination in such a network by (1) having the individual femtocells randomly choosing their channels, (2) preventing a single femtocell from exclusively using all of the capacity of the randomly-selected channel, and (3) having the femtocells individually adapt their transmission powers and channel selections to maximize the average femtocell network rate while satisfying the macrocell performance requirements.

Second, we analytically formulate the resource (spectrum and power) allocation problem according to the proposed AOCA approach as a non-linear program in order to derive the optimal values of its parameters. The solution of such a program provides major insights on the effects of different system parameters, such as the macrocell user density and femtocell network density, the macrocell user performance requirements, and the number/frequency of the macrocell frequency bands, on the performance of the proposed framework.

Finally, we use simulation results to show that the AOCA approach results in multiple folds improvement in the average femtocell rate compared to coordinated frequency planning. Such a significant performance gain is attributed to the probabilistic and non-greedy access mechanism of the proposed approach that (1) allows the femtocells to exploit the spectral opportunities that are typically unexploited by the deterministic coordinated frequency planning (which limits each femtocell to use a single channel), and (2) allows multiple neighbouring femtocells to simultaneously use a given channel without explicit coordination, which increases the femtocell network-wide goodput performance. As the load/denisty of both networks approach the spectrum saturation level, centralized frequency planning achieves higher rates compared to our approach. However, such centralized frequency planning is typically infeasible given the uncontrolled and ad hoc nature of femtocell deployment. 
The remainder of the paper is organized as follows. We define the system model and formulate the opportunistic spectrum access problem in Section II. The proposed AOCA approach is presented in Section III. Then we analytically optimize its parameters and evaluate its performance in Section IV and Section V, respectively. We conclude in Section VI.

\section{System Model and Problem Formulation}

We consider the downlink of a two-tier network that consists of a single macrocell that serves a circular area of radius $R$, within which $N$ femtocells are randomly deployed by home and office users. The macrocell provides cellular access to $M$ randomly located macrocell users (MUs). The random distribution of both the MUs and the femtocells within the macrocell service area are modeled by homogeneous spatial Poisson point processes [1] with densities $\rho_{M U}$ and $\rho_{F}$, respectively. The femtocells share the macrocell spectrum that is divided into $S$ non-overlapping channels (e.g., a channel can refer a component carrier or a resource block in LTE systems). We model the macrocell transmission activities carried over each channel as an ON/OFF source. We define the activity factor of the $i^{t h}$ channel, $a_{M}^{(i)}$, as the fraction of time channel $i$ is carrying MU traffic. A femtocell can access one channel at any given time to serve its associated femtocell user (FU). We assume a single FU per femtocell. Such an assumption is common in the literature due to the opportunistic scheduling operation in practice (which dedicates a channel to the user with the best response) and does not violate the generality of the model [8]. We also model the femtocell activity as an ON/OFF source with activity factor $a_{F}$. Without loss of generality, we assume the activity factor of femtocells $a_{F}$ is fixed throughout the network regardless the used frequency. We do not assume any kind of information exchange whatsoever between the femtocells and the macrocell nor between femtocells amongst each other.

\section{A. Interference Model}

For a given macrocell service area, we assume that the interference from neighbouring macrocells is negligible due to either explicit frequency planning or the use of intercell interference coordination (ICIC) schemes. Hence, the interference scenarios in the system at hand are limited to the following scenarios depicted in Figure 1:

Macrocell-to-femtocell interference: The received interference power from the macrocell base station at an FU operating over channel $i$ is

$$
P_{M-F}^{(i)}=P_{M_{o}}^{(i)}\left(\frac{d}{d_{o}^{(i)}}\right)^{-\alpha} \gamma^{(i)}, \quad d \geq d_{o}^{(i)}
$$

where $d$ is the distance between the macrocell and the $\mathrm{FU}, d_{o}^{(i)}$ is the close in distance that is in the order of the operating wavelength (i.e., few centimeters), $\alpha$ is the path loss exponent of the environment, $P_{M_{o}}^{(i)}=\frac{P_{M}^{(i)} G_{t}^{(i)} G_{r}^{(i)} \lambda_{i}^{2}}{\left(4 \pi d_{o}^{(i)}\right)^{2}}$ is the received power at the close in distance $d_{o}^{(i)}$ where $P_{M}^{(i)}$ is the macrocell transmission power, $G_{t}^{(i)}$ and $G_{r}^{(i)}$ are the transmit and receive antenna gains, respectively, and $\lambda_{i}$ is the channel wavelength.

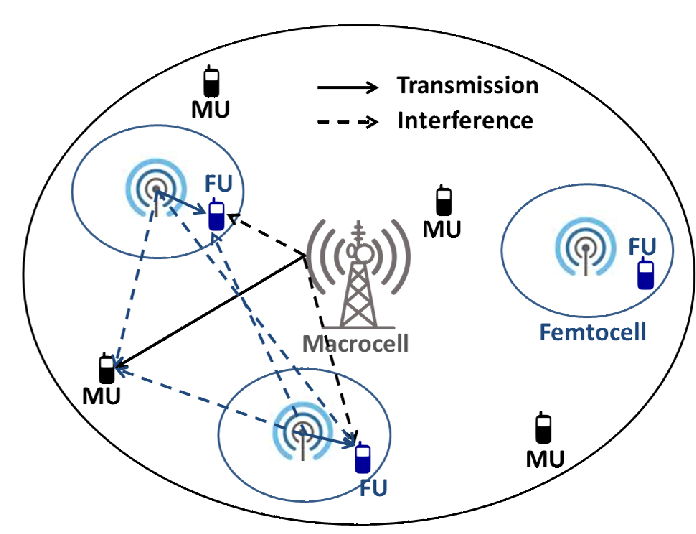

Fig. 1. Interference scenario in two-tier femtocell networks.

For the assumed Rayleigh fading model, the normalized power gain of the fading process $\gamma^{(i)}$ is exponentially distributed.

Femtocell-to-femtocell and femtocell-to-macrocell interference: These two interference scenarios represent the cumulative interference from all of the femtocells using channel $i$ at a certain $\mathrm{FU}$ and $\mathrm{MU}$, respectively. The two interference scenarios have the same model as they are originated by the same source: the femtocell network. However, they represent the interference at different types of users. For a tagged node, either a $\mathrm{FU}$ or a $\mathrm{MU}$, the cumulative received interference power from all the interfering femtocells, $P_{i n t}^{(i)}$, is the sum of the $L$ i.i.d. random variables $P_{l}^{(i)}$, i.e.,

$$
P_{i n t}^{(i)}=P_{1}^{(i)}+P_{2}^{(i)}+\ldots+P_{L}^{(i)}
$$

where $P_{l}^{(i)}$ is the received interference from the $l^{\text {th }}$ femtocell computed similar to (1) with $P_{M_{o}}^{(i)}$ is replaced by $P_{F_{o}}^{(i)}=$ $\frac{P_{F_{l}}^{(i)} G_{t}^{(i)} G_{r}^{(i)} \lambda_{i}^{2}}{\left(4 \pi d_{o}^{(i)}\right)^{2}}$ as $P_{F_{l}}^{(i)}$ is the $l^{\text {th }}$ femtocell transmission power, and $L$ is a random variable that denotes the number of interfering femtocells. Note that the random variable $L$ is independent of the random variables $P_{l}^{(i)}$. In a highly dense femtocell network, the femtocell distribution is modeled as a homogeneous Poisson process [1]. Hence, the probability of having $l$ interfering femtocells in a circle of radius $R_{i n t}$ and area $\pi R_{i n t}^{2}$ is given by

$$
\operatorname{Prob}[L=l]=\frac{e^{-\rho_{F} \pi R_{i n t}^{2}}\left(\rho_{F} \pi R_{\text {int }}^{2}\right)^{l}}{l !}, \quad l=0,1,2, \ldots
$$

where $R_{\text {int }}$ is the coverage radius of the femtocell which is the distance beyond which the femtocell interference is negligible, i.e., below the receiver sensitivity of the FU and MU units. The distribution of the distance between the FU or MU located at the center of a circular area of radius $R_{i n t}$ and the randomly located interferers within is given by [14]

$$
f_{D}\left(d_{l}\right)=\frac{2 d_{l}}{R_{\text {int }}^{2}}, \quad d_{l} \leq R_{\text {int }}
$$

Using the law of total probability, the probability distribution function of $P_{i n t}^{(i)}$ can be calculated as follows:

$$
\operatorname{Prob}\left[P_{i n t}^{(i)}\right]=\sum_{l=0}^{\infty} \operatorname{Prob}\left[P_{\text {int }}^{(i)} \backslash L=l\right] \operatorname{Prob}[L=1]
$$


The conditional distribution of $P_{i n t}^{(i)}$ is difficult to obtain in closed form. However, we use the analytical approach presented in [15] in which we first compute the characteristic function of the conditional distribution of $P_{i n t}^{(i)}$ then reverse it to compute the unconditional distribution of $P_{i n t}^{(i)}$. We omit the detailed proof due to space limitations. Accordingly, we can approximate the distribution of the femtocell-to-femtocell and the femtocell-to-macrocell interference with lognormal distributions. We compute the mean and variance of such lognormal distributions as:

$E\left[P_{i n t}^{(i)}\right]= \begin{cases}2 \pi a_{F} \rho_{F} P_{F_{o}}^{(i)} d_{o}^{(i)^{2}} e^{-\pi a_{F} \rho_{F} d_{o}^{(i)^{2}}} \ln \frac{R_{i n t}}{d_{o}^{(i)}}, & \alpha=2 \\ \frac{2 \pi a_{F} \rho_{F} P_{F_{o}}^{(i)} d_{o}^{(i)^{2}}}{\alpha-2} e^{-\pi a_{F} \rho_{F} d_{o}^{(i)^{2}}}, & \alpha>2\end{cases}$

and

$$
\operatorname{Var}\left[P_{i n t}^{(i)}\right]=\frac{\pi a_{F} \rho_{F}}{\alpha-1}\left[2 P_{F_{o}}^{(i)} d_{o}^{(i)^{2}} e^{-\pi a_{F} \rho_{F} d_{o}^{(i)^{2}}}\right]^{2}, \quad \alpha \geq 2
$$

respectively. Note that the above distribution of $P_{i n t}^{(i)}$ describes the total interference at any given FU or MU. We shall use the above interference model shown in Figure 1 in our opportunistic spectrum access problem formulation.

\section{B. Opportunistic Spectrum Access Problem Formulation}

In this paper, we consider the opportunistic spectrum access problem that aims at maximizing the average rate of the femtocell network while providing statistical guarantees on the performance of the macrocell users. In our statistical model, we probabilistically guarantee an upper bound on the femtocell interference at the macrocell users. Let $P_{i n t}^{\max }$ and $\beta$ define the maximum permissible interference that can be tolerated from the femtocell network and the maximum allowed outage probability at the MUs, respectively. Let $r_{n}^{(i)}$ denote the rate achieved by the $n^{t h}$ FU over channel $i$ defined as:

$$
r_{n}^{(i)}=a_{F} W_{e f f}^{(i)} \log _{2}\left(1+S I N R_{n}^{(i)}\right)
$$

where $S I N R_{n}^{(i)}$ is the signal to interference plus noise ratio experienced by $n^{t h} \mathrm{FU}$ over channel $i$, and $W_{e f f}^{(i)}$ is the $i^{t h}$ channel effective bandwidth equals to $\eta W^{(i)}$, where $W^{(i)}$ is the bandwidth of channel $i$ and $\eta$ models the bandwidth efficiency of the used modulation and coding scheme. Typical values of $\eta$ in LTE systems lie between 0.5 and 0.7 [16].

The generic constrained opportunistic channel access problem can be formulated as follows:

$$
\begin{array}{lll}
\operatorname{maximize} & \frac{1}{N} \frac{1}{S} \sum_{n=1}^{N} \sum_{i=1}^{S} r_{n}^{(i)}, & n=1,2, \ldots, N \\
\text { subject to } & \operatorname{Prob}\left[P_{\text {int }}^{(i)} \leq P_{\text {int }}^{\max }\right] \leq \beta, \quad i=1,2, \ldots, S \\
& P_{\min } \leq P_{F_{n}}^{(i)} \leq P_{\max }
\end{array}
$$

where $P_{F_{n}}^{(i)}$ is the maximum transmission power of the $n^{\text {th }}$ femtocell, ${ }^{n} P_{\min }$ and $P_{\max }$ are the lower and upper bounds of the femtocell transmission power, respectively. The lower bound $P_{\min }>0$ is to guarantee a minimum rate per femtocell in the worst case. We next introduce our proposed autonomous opportunistic channel access approach then reformulate this generic problem accordingly to optimize its performance.

\section{Autonomous Opportunistic Spectrum ACCESS IN FEMTOCELl Networks}

We propose the Autonomous Opportunistic Channel Access (AOCA) approach that allows the femtocells to access the macrocell channels while statistically guaranteeing an upper bound on the performance of the macrocell users. The main distinguishing feature of the proposed AOCA approach is that it does neither rely on any kind of explicit coordination amongst the femtocells each other nor with the macrocell base station. The proposed OOSA framework has two main components: (1) a randomized channel selection component that addresses the inability to explicitly coordinate the individual channel selections of the femtocells, combined with (2) a non-greedy channel access mechanism which probabilistically enables the femtocells to share the available wireless capacity in a distributed manner without explicit coordination.

\section{A. Randomized Channel Selection}

As we explained earlier, the randomly deployed femtocells are unable to apriori know the channel assignment of their neighbours due to the lack of explicit coordination. To counter such limitations, we propose the following random channel selection approach. A femtocell randomly selects a channel to use from the pool of available channels (if there does not exist a preferred channel that recently carried out successful transmissions). Due to the inability of a femtocell to neither accurately assess the impact of its transmission on nearby ongoing MUs nor know the channel utilization profile of the surrounding femtocells and MUs, a femtocell chooses any channel with equal probability. Hence, we use randomization to spread multiple femtocells over different channels and alleviate the need for explicit inter-femtocell coordination. However, such a randomized channel selection neither ensures the fair sharing of the available capacity between different femtocells nor guarantees certain levels of macrocell users' performance. In order to achieve these goals, we present the following probabilistic transmission scheme that complements such randomized channel selection.

\section{B. Adaptive Probabilistic Transmission}

Recall that a femtocell does not know whether or not its transmission will interfere with any nearby on-going MU receptions nor if other nearby femtocells have also selected the same channel. We propose the following probabilistic channel access mechanism which is conservative and nongreedy in exploiting the randomly selected channel, and hence, it probabilistically reduces MU outages due to miss inferring the existence of nearby MUs. Furthermore, such a probabilistic approach allows multiple femtocells to simultaneously exploit a given spectral opportunity since it allows the femtocell to transmit at the maximum power level that can be used, $P_{F_{n}}^{(i)}$, only with a certain probability $p$. Hence, the AOCA approach probabilistically leaves a capacity margin that can be utilized by other femtocells in the system that happened to simultaneously select the same channel.

On the other hand, the AOCA approach will have the femtocell using a lower power between $P_{\min }$ and $\zeta P_{F_{n}}^{(i)}$, where $0<\zeta<1$, with probability $(1-p)$. While potentially 
degrading the femtocell rate, the use of low power transmission further reduces the probability of intercepting unidentified macrocell transmissions. In addition, it allows multiple neighbouring femtocells to simultaneously use a given channel to increase the aggregate femtocell network rate. Recall that a lower transmission power implies a lower transmission rate realized via a low order modulation scheme which is more robust to interference that cannot be explicitly nulled out [17]. The AOCA protocol realization starts from the minimum transmission power level, $P_{\min }$, and will increase the power used with probability $(1-p)$ until either $\zeta P_{F_{n}}^{(i)}$ is reached or a transmission failure occurs. Such a gradual reduction of the unutilized capacity margin is to not sacrifice the average femtocell rate if there does not exist any nearby MUs on the randomly-selected channel. Meanwhile, if a nearby femtocell uses the same channel, it will cause the high rate transmission to fail. As long as the high power transmissions are successful on the randomly-selected channel, the femtocell declares a channel as its favourite channel. Otherwise, the femtocell will randomly choose a new channel. Algorithm I outlines the AOCA approach.

\section{Analytical Performance Optimization}

In this section, we analyze the proposed AOCA approach in order to derive its optimal parameter values. More specifically, we reformulate the generic constrained opportunistic channel access problem given in (9) to find the values of the probability of high power transmission $p$, the low power margin $\zeta$, and the maximum femtocell transmission powers over different frequency channels $P_{F_{n}}^{(1)}, P_{F_{n}}^{(2)}, \ldots, P_{F_{n}}^{(S)}$ that maximize the average femtocell network rate while providing statistical guarantees on the performance of the macrocell users.

\section{A. Average Femtocell Rate}

According to the AOCA approach, the femtocell uses the highest possible rate (obtained when the femtocell is transmitting at the highest power $P_{F_{n}}^{(i)}$ ) with probability $p$ and a variable lower rate with probability $(1-p)$. Consequently, the FU rate when using channel $i$ can be expressed as

$$
\begin{aligned}
r_{n}^{(i)}=a_{F} W_{\text {eff }}^{(i)} \quad[ & {\left[p \log _{2}\left(1+S I N R_{n}^{(i)}\right)\right.} \\
& \left.+(1-p) \log _{2}\left(1+\zeta S I N R_{n}^{(i)}\right)\right]
\end{aligned}
$$

where $S I N R_{n}^{(i)}$ is the received signal to interference plus noise ratio of the femtocell that can be expressed as

$$
\operatorname{SINR}_{n}^{(i)}=\frac{P_{F_{n_{o}}}^{(i)}\left(\frac{d_{F}}{d_{o}^{(i)}}\right)^{-\alpha} \gamma^{(i)}}{W^{(i)} N_{o}+a_{M}^{(i)} P_{M_{o}}^{(i)}\left(\frac{d_{M}}{d_{o}^{(i)}}\right)^{-\alpha} \gamma^{(i)}+P_{i n t}^{(i)}}
$$

where $d_{F}$ and $d_{M}$ are the distances between the tagged FU and the femtocell and macrocell base stations, respectively, and $N_{o}$ is the power spectral density of the white Gaussian noise. Note that we do not incorporate the ramp up from the minimum possible rate to the rate obtained at the steady state power $\zeta P_{F_{n}}^{(i)}$ in our formulation. While such an assumption slightly impacts the FU achievable rate, it does not affect our optimization problem as the maximum interference constraints depend only on the maximum used power, $P_{F_{n}}^{(i)}$, and $\zeta$.

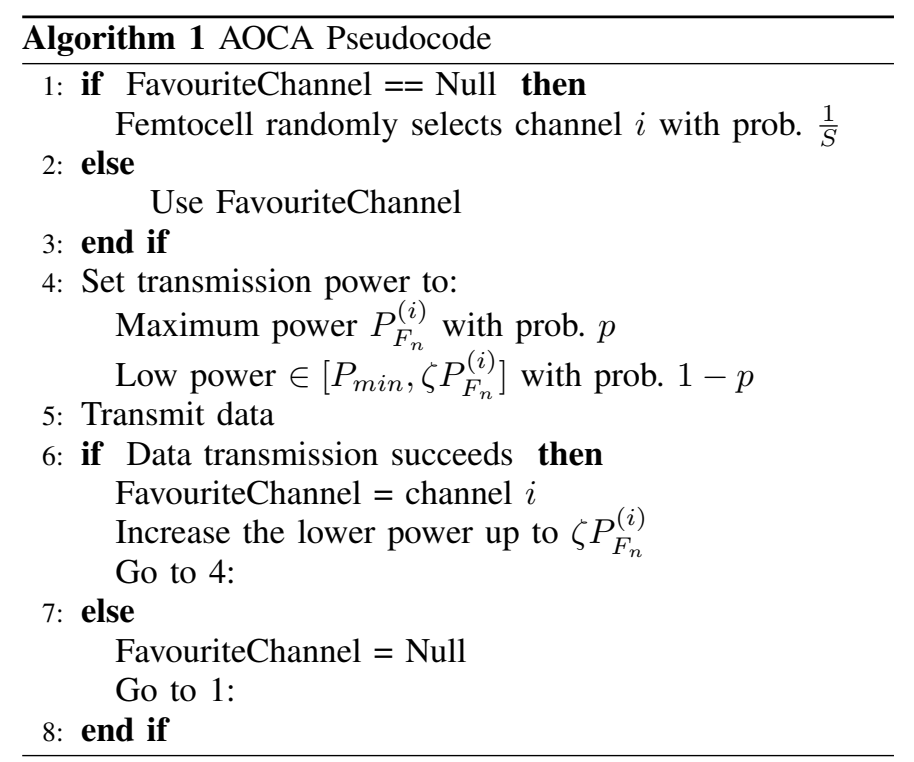

\section{B. Macrocell Statistical Performance Guarantees}

Since we have approximated $P_{i n t}^{(i)}$ with a lognormal distribution, the probability that $P_{i n t}^{(i)}$ does not exceed a certain margin $P_{i n t}^{\max }$ is given by

$$
\operatorname{Prob}\left[P_{\text {int }}^{(i)} \leq P_{\text {int }}^{\max }\right]=\frac{1}{2} \operatorname{erfc}\left(\frac{\ln P_{\text {int }}^{\max }-\mu}{\sqrt{2 \sigma^{2}}}\right)
$$

where

$$
\begin{aligned}
\mu & =\ln \left(E\left[P_{i n t}^{(i)}\right]\right)-\frac{1}{2} \ln \left(1+\frac{\operatorname{Var}\left[P_{i n t}^{(i)}\right]}{E\left[P_{i n t}^{(i)}\right]^{2}}\right) \\
& =\ln \left(\frac{2 \pi a_{F} \rho_{F} P_{F_{l_{o}}}^{(i)} d_{o}^{(i)^{2}}}{\alpha-2} e^{\pi a_{F} \rho_{F} d_{o}^{(i)^{2}}}\right) \\
& -\frac{1}{2} \ln \left(1+\frac{(\alpha-2)^{2}}{(\alpha-1) \pi a_{F} \rho_{F}}\right)
\end{aligned}
$$

and

$$
\sigma^{2}=\ln \left(1+\frac{\operatorname{Var}\left[P_{i n t}^{(i)}\right]}{E\left[P_{i n t}^{(i)}\right]^{2}}\right)=\ln \left(1+\frac{(\alpha-2)^{2}}{(\alpha-1) \pi a_{F} \rho_{F}}\right)
$$

Note that while $\mu$ depends logarithmically on the femtocell maximum transmission power through $P_{F_{l_{o}}}^{(i)}, \sigma^{2}$ is independent of the value of the femtocell transmission power. Hence, the constraint that $\operatorname{Prob}\left[P_{i n t}^{(i)} \leq P_{\text {int }}^{\max }\right] \leq \beta$ can be formulated in terms of the femtocell transmission power as

$$
P_{F_{n}}^{(i)}<\frac{2^{\ln P_{i n t}^{\max }+\frac{\sigma^{2}}{2}-\sqrt{2 \sigma^{2}} e r f c^{-1}(2 \beta)}}{\frac{2 \pi a_{F} \rho_{F} d_{o}^{(i)^{2}}}{\alpha-2} e^{\pi a_{F} \rho_{F} d_{o}^{(i)^{2}}}} \frac{\left(4 \pi d_{o}^{(i)}\right)^{2}}{G_{t}^{(i)} G_{r}^{(i)} \lambda_{i}^{2}}
$$

Subsisting in (9), the AOCA constrained opportunistic spectrum access problem can be stated as 
TABLE I

SiMUlation PARAMETERS

$$
\begin{array}{ll}
\text { maximize } & \frac{1}{N} \frac{1}{S} \sum_{n=1}^{N} \sum_{i=1}^{S} r_{n}^{(i)}, \quad n=1,2, \ldots, N \\
\text { subject to } & \frac{P_{\min }}{P_{\max }}<\zeta<1, \quad i=1,2, \ldots, S \\
& 0 \leq p \leq 1 \\
& P_{\min } \leq P_{F_{n}}^{(i)} \leq P_{\max } \\
& P_{F_{n}}^{(i)}<\frac{2^{\ln P_{i n t}^{\max }+\frac{\sigma^{2}}{2}-\sqrt{2 \sigma^{2}} e r f c^{-1}(2 \beta)}}{\frac{2 \pi a_{F} \rho_{F} d_{o}^{(i)^{2}}}{\alpha-2}} e^{\pi a_{F} \rho_{F} d_{o}^{(i)^{2}}} \frac{\left(4 \pi d_{o}^{(i)}\right)^{2}}{G_{t}^{(i)} G_{r}^{(i)} \lambda_{i}^{2}}
\end{array}
$$

Solving this non-linear optimization problem off-line for a given system parameters, we obtain the AOCA $p$ and $\zeta$ values alongside the maximum powers $P_{F_{n}}^{(1)}, P_{F_{n}}^{(2)}, \ldots, P_{F_{n}}^{(S)}$ to be used by the different femtocells in order to maximize the average femtocell network rate while providing a statistical guarantee $\beta$ on the interference caused at the MUs, $P_{\text {int }}^{\max }$.

\section{Performance Evaluation}

Here, we evaluate the performance of the proposed AOCA approach via MATLAB simulations. We consider a single macrocell with a 500 meters coverage radius with 4 frequency channels, each of bandwidth $10 \mathrm{MHz}$. The MUs and femtocells are distributed within the macrocell coverage area according to homogeneous spatial Poisson point processes. While the MU densities per channel are simulation variables, we set the femtocell density to its maximum value of 625 femtocells $/ \mathrm{km}^{2}$ for a 20 meters femtocell coverage radius. We consider tight MU statistical guarantees: $P_{\text {int }}^{\max }=-67 \mathrm{dBm}$ and $\beta=1 \%$. The simulations parameters are listed in Table I.

Our performance benchmark is a coordinated channel access approach that exploits centralized frequency planning to eliminate the femtocell-to-femtocell interference based on the availability of the global network-wide information. Given the coordinated channel allocation of such a frequency plan, we solve the generic constrained femtocell performance optimization problem given in (9) to compute the maximum femtocell transmission powers satisfying the MU performance requirement. Such a benchmark represents the upper bound of the performance of the wide set of existing schemes that assume explicit coordination within femtocell network (e.g., explicit interference coordination schemes) and/or coordination with the macrocell. We compare the performance of our proposed autonomous probabilistic approach (which allows a femtocell to opportunistically exploit the entire macrocell spectrum) against such a coordinated deterministic approach (which allocates a single channel per femtocell) in order to demonstrate the AOCA performance gains despite the absence of any kind of coordination in such a two-tier network.

\section{A. Impact of Macrocell users and Femtocell densities}

In this section, we study the impact of the macrocell (assuming equal activity factor for all MUs) and femtocell densities/loads on the AOCA performance. At low MU densities, the average femtocell rate linearly increases with the femtocell activity as the case with coordinated access due to the absence of significant femtocell interference. However,

\begin{tabular}{|c|c|}
\hline Parameter & Value \\
\hline \hline Path loss exponent $(\alpha)$ & 4 \\
\hline Number of channels $(S)$ & 4 \\
\hline Channel bandwidth & $10 \mathrm{MHz}$ \\
\hline AWGN power density $\left(N_{o}\right)$ & $-160 \mathrm{dBm} / \mathrm{Hz}$ \\
\hline Maximum allowed interference $P_{\text {int }}^{\text {max }}$ & $-67 \mathrm{dBm}$ \\
\hline Statistical MU outage guarantee $(\beta)$ & $1 \%$ \\
\hline Transmit and receive antenna gains $\left(G_{t}, G_{r}\right)$ & $0 \mathrm{~dB}$ \\
\hline Macrocell radius & $500 \mathrm{~m}$ \\
\hline Macrocell power & $20 \mathrm{~W}$ \\
\hline Femtocell radius & $20 \mathrm{~m}$ \\
\hline Maximum femtocell power $\left(P_{\max }\right)$ & $20 \mathrm{~mW}$ \\
\hline Minimum femtocell power $\left(P_{\min }\right)$ & $1.8 \mathrm{~mW}$ \\
\hline Femtocell bandwidth efficiency $(\eta)$ & $0.5 \mathrm{~m}$ \\
\hline
\end{tabular}

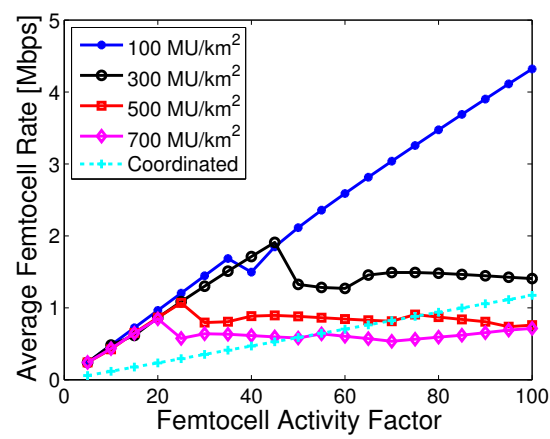

Fig. 2. Average femtocell rate increases with the femtocell activity factors for different per channel MU densities.

the AOCA average femtocell rate is multiple folds of the benchmark rate (e.g., Figure 2 shows up to $310 \%$ gain). Such a gain doubles when the femtocell density decreases. Recall that, these results are for the maximum femtocell density.

As the MU density increases, the AOCA gain decreases. Furthermore, the average AOCA femtocell rate tends to saturate with the increase of the femtocell activity. Such a decrement in the average AOCA rate with the increase of either the femtocell activity or the macrocell density is attributed to the reduction in the AOCA transmission parameters $p$ and $\zeta$ as well as the femtocell transmission powers satisfying the MU performance constraint. Hence, coordinated access outperforms the proposed scheme when the system if fully loaded. However, coordinated frequency planning is based upon unrealistic system assumptions unlike the AOCA approach that does not assume cooperation neither between the femtocells each other nor with the macrocell.

Next, we vary the activity of the MUs. While the AOCA gain exhibits similar trends for various MU activity patterns, the gain increases with the increase in the MU activities despite the reduction in the average femtocell rate.

\section{B. Impact of Macrocell Performance Constraints}

We evaluate the impact of the MU performance constraints over the AOCA parameters. Namely, we study how the maximum allowed interference limit, $P_{i n t}^{\max }$, and the statistical outage constraint, $\beta$, affect the optimal AOCA parameter values. The maximum femtocell transmission power according to both AOCA and the coordinated frequency planning increases with the relaxation of the MU constraints (i.e., higher 


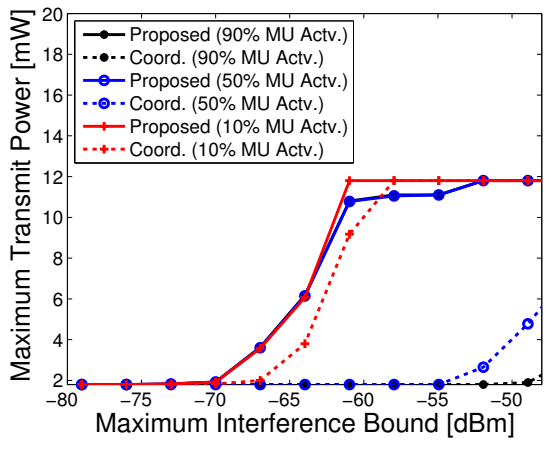

Fig. 3. AOCA probabilistic access mechanism allows the femtocell to use higher power compared to the coordinated access approach.

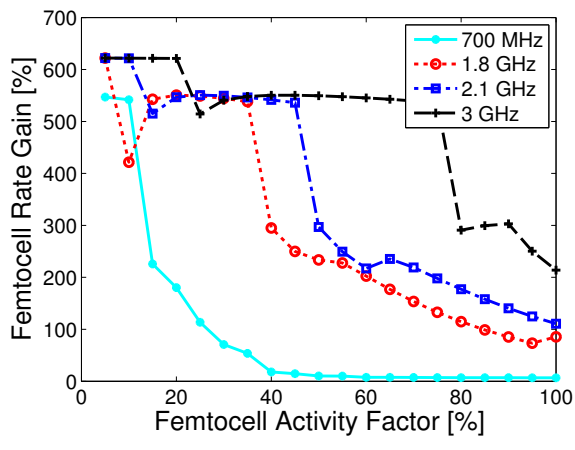

Fig. 4. AOCA gain increases with the operating frequency of the band to which all 4 channels belong to for $300 \mathrm{MU} / \mathrm{km}^{2}$ and $90 \%$ MU activity.

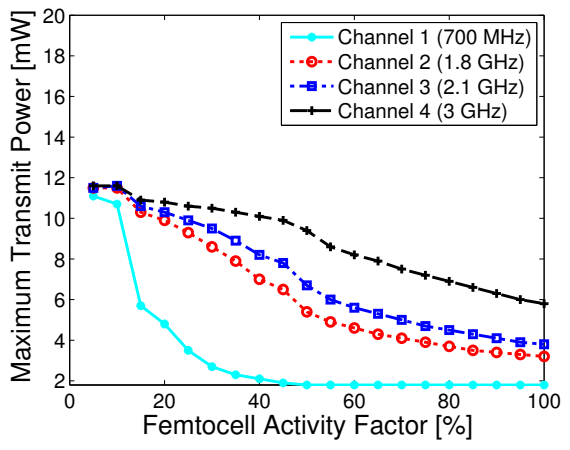

Fig. 5. The AOCA approach allocates more power to channels with higher frequency when the channels belong to different frequency bands.
$P_{\text {int }}^{\max }$ or $\beta$ values). However, the AOCA approach allows the femtocells to use higher power compared to the coordinated access approach as shown in Figure 3. This is attributed to the AOCA probabilistic transmission mechanism that allows the femtocell to use the maximum power only with probability $p$.

\section{Impact of Operating Frequency}

Finally, we assess the impact of the operating frequency. We study the cases in which (1) all the channels belong to the same LTE band and vary the band frequency, and (2) each of the $S$ channels belongs to a different band. We consider the $700 \mathrm{MHz}, 1.8 \mathrm{GHz}, 2.1 \mathrm{GHz}$, and the $3 \mathrm{GHz}$ LTE bands. In both cases, the AOCA gain increases with the operating frequency regardless of the MU density or activity pattern (e.g., see Figure 4). Such a behavior is attributed to the better propagation characteristics of lower frequencies that extends the transmission range for a given transmission power. Consequently, the number of the femtocells that can simultaneously share a spectrum due to AOCA decreases with the decrease of the operating frequency, and hence, the AOCA gain decreases due to the reduction in the values $p$ and $\zeta$ as well as the maximum femtocell powers obtained by solving (16). Figure 5 depicts the maximum power allocated to different channels in the latter case in which each channel belongs to a different band. While we only present the results for $S$ equals to 4 , similar performance trends were obtained for different $S \in[1,5]$. The only impact of the increase in $S$ is an increase of the AOCA gain.

\section{CONCLUSIONS}

In this paper, we have presented the autonomous opportunistic channel access framework. The framework allows the randomly deployed femtocell network to share the channels available to the overlaying macrocell network without any kind of coordination between neither the femtocells amongst each other nor with the macrocell base station. The proposed AOCA approach adopts probabilistic channel access mechanism that allows the femtocells to exploit the spectral opportunities in a way that maximizes the average femtocell rate while providing statistical guarantees on the performance experienced at the macrocell users. Simulation results have shown that the proposed approach achieves multi-fold improvement in the average femtocell rate gain when the macrocell offered load does not saturate the capacity of the available channels.

\section{REFERENCES}

[1] V. Chandrasekhar, J. G. Andrews, and A. Gatherer, "Femtocell networks: a survey," IEEE Communications Mag., vol. 46, pp. 59-67, Sep. 2008.

[2] H. Claussen, "Performance of macro- and co-channel femtocells in a hierarchical cell structure," in Proc. of IEEE PIMRC07, Athens, Greece, Sep. 2007.

[3] L. Garcia, K. Pedersen, and P. Mogensen, "Autonomous component carrier selection: Interference management in local area environments for LTE-advanced," IEEE Communications Mag., vol. 47, no. 9, pp. 110-116, Sep. 2009.

[4] F. Sanchez-Moya, J. Villalba-Espinosa, L. G. U. Garcia, K. I. Pedersen, and P. E. Mogensen, "On the impact of explicit uplink information on autonomous component carrier selection for LTE-A femtocells," in Proc. of IEEE VTC 2011-Spring, Budapest, Hungary, May 2011.

[5] L. G. U. Garcia, I. Z. Kovacs, K. I. Pedersen, G. W. O. Costa, and P. E. Mogensen, "Autonomous component carrier selection for $4 \mathrm{G}$ femtocells - a fresh look at an old problem," IEEE Journal on Sel. Areas in Comm., vol. 30, no. 3, pp. 525-537, Apr. 2012.

[6] V. Chandrasekhar and J. G. Andrews, "Spectrum allocation in tiered cellular networks," IEEE Trans. on Communications, vol. 57, no. 10, pp. 3059-3068, Oct. 2009.

[7] X. Kang, R. Zhang, and M. Motani, "Price-based resource allocation for spectrum-sharing femtocell networks: A stackelberg game approach," IEEE Journal of Sel. Areas in Comm., vol. 30, no. 3, pp. 538-549, Apr. 2012.

[8] V. Chandrasekhar, J. G. Andrews, T. Muharemovic, Z. Shen, and A. Gatherer, "Power control in two-tier femtocell networks," IEEE Wireless Communications, vol. 8, no. 8, pp. 4316-4328, Aug. 2009.

[9] H.-S. Jo, C. Mun, J. Moon, and J.-G. Yook, "Interference mitigation using uplink power control for two-tier femtocell networks," IEEE Trans. on Wireless Communications, vol. 8, no. 10, pp. 4906-4910, Oct. 2009.

[10] S. Rangan and R. Madan, "Belief propagation methods for intercell interference coordination in femtocell networks," IEEE Journal on Sel. Areas in Comm., vol. 30, no. 3, pp. 631-640, Apr. 2012.

[11] O. N. Gharehshiran, A. Attar, and V. Krishnamurthy, "Collaborative subchannel allocation in cognitive LTE femto-cells: A cooperative gametheoretic approach," IEEE Trans. on Communications, vol. 16, no. 1, pp. 325-334, Jan. 2013.

[12] M. Rasti, A. R. Sharafat, and B. Seyfe, "Pareto-efficient and goal driven power control in wireless networks: A game-theoretic approach with a novel pricing scheme," IEEE/ACM Trans. on Networking, vol. 17, no. 2, pp. 556-569, Apr. 2009.

[13] S. Ren, J. Park, and M. Schaar, "Entry and spectrum sharing scheme selection in femtocell communications markets," IEEE/ACM Trans. on Networking, vol. 21, no. 1, pp. 218-232, Feb. 2013.

[14] E. Sousa and J. Silvester, "Optimum transmission ranges in a directsequence spread-spectrum multihop packet radio network," IEEE Journal of Sel. Areas in Comm., vol. 8, no. 5, pp. 762-771, Jun. 1990.

[15] H. B. Salameh, M. Krunz, , and O. Younis, "MAC protocol for opportunistic cognitive radio networks with soft guarantees," IEEE Trans. on Mobile Computing, vol. 8, no. 10, pp. 1339-1352, Oct. 2009.

[16] P. Mogensen, W. Na, I. Kovacs, F. Frederiksen, A. Pokhariyal, K. Pedersen, T. Kolding, K. Hugl, and M. Kuusela, "LTE capacity compared to the shannon bound," in Proc. of IEEE VTC 2007-Spring, Dublin, Ireland, Apr. 2007.

[17] T. Rappaport, Wireless Communications, Principles \& Practice.Prentice Hall, 1996. 